\title{
Use of Low-Cost Videomicroscopy versus Standard Videodermatoscopy in Trichoscopy: A Controlled, Blinded Noninferiority Trial
}

\author{
Anna Elisa Verzì Francesco Lacarrubba Giuseppe Micali \\ Dermatology Clinic, University of Catania, Catania, Italy
}

\section{Key Words}

Alopecia - Alopecia areata - Androgenic alopecia .

Dermoscopy $\cdot$ Lichen planopilaris · Trichoscopy

\begin{abstract}
Affordable (USD 30) handheld USB digital microscopes, or videomicroscopes (VMs), that provide $\times 10-200$ magnification are available on the web for nonmedical uses such as in botany, entomology, microelectronics or, recently, for hair/ scalp evaluation. The aim of this study was to compare the reliability of low-cost VMs versus standard, medically marketed videodermatoscopes (VDs) in trichoscopy. Twentyfive patients affected by different types of hair loss were enrolled in a controlled, blinded noninferiority trial. All patients underwent examination by two low-cost VMs as well as by standard VD in order to evaluate any variability in the detection of common trichoscopic features. At the end of the study, the two low-cost VMs enabled a correct identification of all hair shaft alterations; as regards follicular and/or perifollicular examination, black dots were easily recognized by both equipments, but other follicular features, such as yellow dots, white dots and perifollicular scales, were not always adequately visualized because of low color quality and/or reduced brightness and/or resolution. In conclusion, our study suggests that the potential accuracy of low-cost
\end{abstract}

VMs in the evaluation of hair loss may have some pitfalls. Therefore, a low-cost VM should not be routinely used for reliable scalp trichoscopy, unless supported by individual controlled noninferiority trials.

(c) 2016 S. Karger AG, Basel

\section{Introduction}

Videodermatoscopy is a noninvasive diagnostic tool that allows a rapid in vivo observation at high magnifications (up to $\times 1,000$ ) of some skin features invisible to the naked eye. The images obtained by a high-resolution videodermatoscope (VD) may be visualized on a monitor, processed and/or stored [1]. Videodermatoscopy is utilized both in the differential diagnosis of pigmented lesions as well as in other fields of dermatology such as inflammatory disorders, ectoparasitoses, other infectious diseases as well as hair and nail abnormalities [1-3]. The evaluation of hair and scalp disorders by videodermatoscopy has recently been defined as trichoscopy [4-6]. In general, the magnification needed to perform a trichoscopic exam ranges from $\times 20$ to $\times 200$, while standard handheld dermatoscopes are equipped with lenses up to $\times 10$; therefore, high-magnification systems are required. Videomicroscopes (VMs) are simple, affordable hand-

\section{KARGER 125\%}

(c) 2016 S. Karger AG, Base

2296-9195/16/0014-0172\$39.50/0

E-Mail karger@karger.com

www.karger.com/sad
Giuseppe Micali, MD

Dermatology Clinic, University of Catania

A.O.U. Policlinico-Vittorio Emanuele, Via Santa Sofia, 78

IT-95123 Catania (Italy)

E-Mail cldermct@gmail.com 
Fig. 1. Package (a) and brochure (b) of the two VMs used in the study; both claim the use in hair/scalp evaluation.

Fig. 2. Lichen planopilaris (a-c) and alopecia areata $(\mathbf{d}-\mathbf{f})$. Perifollicular scales typical of lichen planopilaris are well visible using the standard VD (a), but not using VM 1 (b) and VM 2 (c). Yellow dots typical of alopecia areata are well identifiable using both standard VD (d) and VM 1 (e), but not using VM 2 (f).
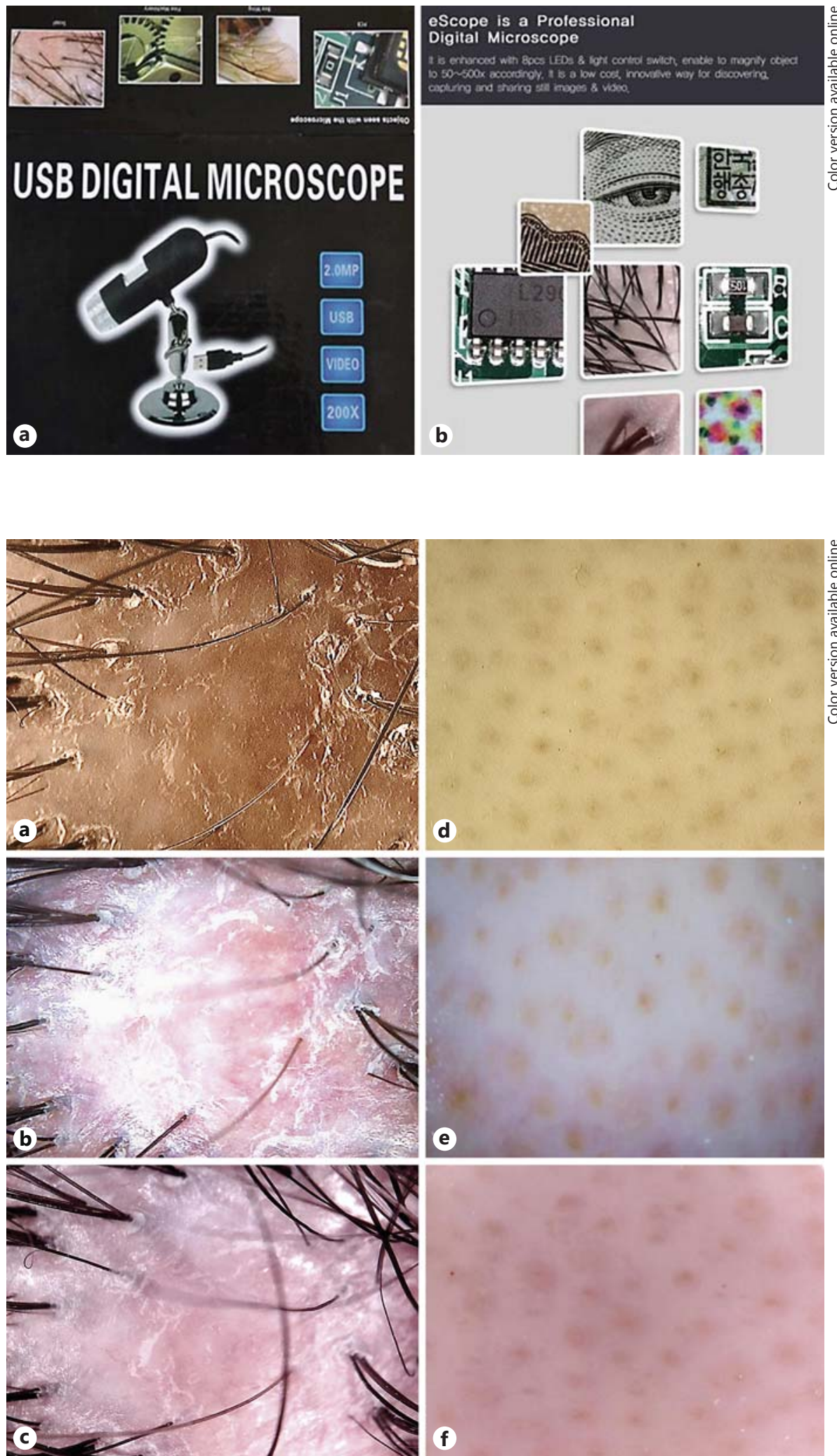

e

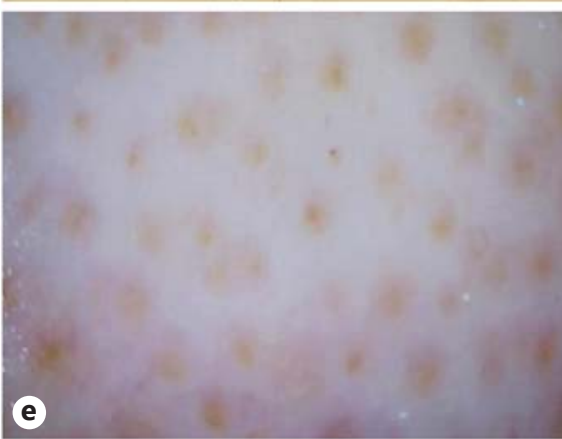

f 
held USB digital microscopes that provide magnification ranging from $\times 10$ to $\times 200$. They are available on the web for various nonmedical uses such as in botany, entomology, microelectronics and, recently, for hair/scalp evaluation (fig. 1). In a previous study, we have demonstrated that some VMs allowed a definitive diagnosis of scabies, identifying at low magnification the burrow and at high magnification other typical signs such as mites, eggs and feces. These results were similar to a medically marketed $\mathrm{VD}$, although the latter provided a slightly better image definition [7]. We concluded that where noninvasive diagnostic techniques are crucial to control scabies such as in some institutional settings and/or geographic areas of unmet need, the impact of VMs appears to be significant and cost-effective. Based on these considerations, we have performed a clinical study to compare the reliability of two low-cost VMs with a standard, medically marketed VD in the differential diagnosis of hair loss.

\section{Methods}

Twenty-five patients (male: 8, female: 17; age range: 18-64 years) affected by different types of hair loss including 10 cases of alopecia areata, 8 of androgenetic alopecia, 3 respectively of lichen planopilaris and frontal fibrosing alopecia, and 1 of trichotillomania were enrolled in a controlled, blinded noninferiority clinical trial. All patients underwent examination by two low-cost VMs (VM 1: Micron Technology USB-Microscope; VM 2: Oitez eScope, approximately USD 30 each) as well as by a standard VD at incident light (Hirox KH-1300, approximately USD 20,000) in order to evaluate any variability in the detection of common trichoscopic features localized in the hair shaft (hair diameter diversity, miniaturized hairs, exclamation mark hairs and broken hairs) and in the follicular (yellow, white and black dots) and perifollicular (perifollicular scales) areas. Three different fields were examined for each patient. In case of follicular examination, the epiluminescence technique (i.e. the application of oil between the lens and the skin to avoid light reflection) was performed, while hair shaft and perifollicular features were evaluated by a 'dry' trichoscopy (without the use of oil). Images obtained by the three systems ( $\times 30$ magnification) were independently evaluated by three dermatologists blinded to the system adopted. three experts were identical. As expected, the standard medically marketed VD allowed for an optimal visualization of all hair shaft, follicular and perifollicular features. As regards the two low-cost VMs, they enabled a correct identification of all hair shaft alterations; as regards the

follicular and/or perifollicular examination, black dots were easily recognized by both equipments, but other follicular features, such as yellow dots with VM 2 and white dots and perifollicular scales with both VM 1 and 2, were not adequately visualized because of low color quality and/or reduced brightness and/or resolution (fig. 2).

\section{Conclusions}

According to our study, VMs have a limited role in the assessment of hair disorders compared to standard VDs. However, further controlled noninferiority trials are needed to support our observations since the present study assessed only two types of VMs and was performed on a restricted number of patients, thus not allowing high-quality evidence-based conclusions.

\section{Statement of Ethics}

All subjects provided informed consent to their participation in the study. Institutional review board approval was waived by the local ethics committee.

\section{Disclosure Statement}

The authors declare that they have no conflicts of interest.

\section{Results}

At the end of the study, the results obtained by the

\section{References}

1 Lacarrubba F, Dall'Oglio F, Nasca MR, Micali G: Videodermatoscopy enhances diagnostic capability in some forms of hair loss. Am J Clin Dermatol 2004;5:205-208.

2 Micali G, Lacarrubba F, Massimino D, Schwartz RA: Dermatoscopy: alternative uses in daily clinical practice. J Am Acad Dermatol 2011;64:1135-1146.

3 Miteva M, Tosti A: Hair and scalp dermatoscopy. J Am Acad Dermatol 2012;67:1040-1048.

4 Lacarrubba F, Micali G, Tosti A: Scalp dermoscopy or trichoscopy. Curr Probl Dermatol (Switzerland) 2015;47:21-32.

5 Rudnicka L, Olszewska M, Rakowska A, Slowinska M: Trichoscopy update 2011. J Dermatol Case Rep 2011;5:82-88.

6 Jain N, Doshi B, Khopkar U: Trichoscopy in alopecias: diagnosis simplified. Int J Trichology 2013;5:170-178.

7 Micali G, Lacarrubba F, Verzì AE, Nasca MR Low-cost equipment for diagnosis and management of endemic scabies outbreaks in underserved populations. Clin Infect Dis 2015; 60:327-329. 